\title{
ON THE MAXIMUM OF A NORMAL STATIONARY STOCHASTIC PROCESS ${ }^{1}$
}

\author{
BY HARALD CRAMÉR
}

Communicated by W. Feller, May 1, 1962

1. Let $x(t)$ with $-\infty<t<+\infty$ be the variables of a real, separable, normal and stationary stochastic process, such that $E[x(t)]=0$ and $E\left[x^{2}(t)\right]=1$. Let the covariance function of the process be

$$
r(t)=E[x(t) x(0)]=\int_{0}^{\infty} \cos \lambda t f(\lambda) d \lambda,
$$

and assume that the spectral density $f(\lambda)$ is of bounded variation in $(-\infty, \infty)$ and satisfies the condition

$$
\int_{0}^{\infty} \lambda^{2}(\log (1+\lambda)) a f(\lambda) d \lambda<\infty
$$

for some $a>1$.

Then it is known (Hunt [5], Belayev [1]) that the sample functions $x(t)$ will almost certainly be everywhere continuous and have continuous first derivatives $x^{\prime}(t)$. Consequently for every fixed $t>0$ the maximum

$$
\max _{0 \leqq u \leqq t} x(u)
$$

will be a random variable defined but for equivalence.

For the sake of typographical convenience, we write in the sequel simply $\max x(u)$, omitting the subscript $0 \leqq u \leqq t$, and similarly in respect of $\min x(u)$.

The object of this note is to prove the relation

$$
\lim _{t \rightarrow \infty} P\left[\left|\max x(u)-(2 \log t)^{1 / 2}\right|<\frac{\log \log t}{(\log t)^{1 / 2}}\right]=1 .
$$

The notation $P[\cdots]$ denotes here, as throughout the sequel, the probability of the relation between the brackets.

A similar relation was recently given for the case of a normal stationary sequence $x_{n}$ with $n=0, \pm 1, \cdots$ by Berman [2].

2. We shall first prove that

${ }^{1}$ Research work done (Tech. Report No. 1) partially under Contract NASw-334, National Aeronautics and Space Administration. 


$$
P\left[\max x(u) \leqq(2 \log t)^{1 / 2}-\frac{\log \log t}{(\log t)^{1 / 2}}\right] \rightarrow 0
$$

as $t \rightarrow \infty$.

Let $c>0$ be given, and define a random variable $y(u)$ by writing for any real $u$

$$
y(u)= \begin{cases}1 & \text { if } x(u)>c, \\ 0 & \text { if } x(u) \leqq c .\end{cases}
$$

Then $y(u)$ will define a stationary process such that

$$
\begin{aligned}
E[y(u)] & =P[x(u)>c]=\int_{0}^{\infty} \phi(x) d x, \\
E[y(u) y(v)] & =P[x(u)>c, x(v)>c] \\
& =\int_{c}^{\infty} \int_{c}^{\infty} \phi(x, y ; r) d x d y,
\end{aligned}
$$

where

$$
\begin{aligned}
\phi(x) & =\frac{1}{(2 \pi)^{1 / 2}} \exp \left(-\frac{x^{2}}{2}\right), \\
\phi(x, y ; r) & =\frac{1}{2 \pi\left(1-r^{2}\right)^{1 / 2}} \exp \left(-\frac{x^{2}-2 r x y+y^{2}}{2\left(1-r^{2}\right)}\right), \\
r & =r(u-v) .
\end{aligned}
$$

It follows (cf. e.g. Loève [6, pp. 472, 520]) that the integral

$$
z(l)=\int_{0}^{t} y(u) d u
$$

is defined both in quadratic mean and as a sample function integral, and that the two integrals coincide, but for equivalence. Then $z(t)$ will, with probability 1 , be equal to the Lebesgue measure of the set of points $u$ in $[0, t]$ such that $x(u)>c$. Thus $z(t) \geqq 0$ with probability 1 , and

$$
P[z(t)=0]=P[\max x(u) \leqq c] .
$$

For all sufficiently large $c$ we have (Loève, l.c.)

$$
E[z(t)]=t \int_{c}^{\infty} \phi(x) d x>\frac{t}{3 c} \exp \left(-\frac{c^{2}}{2}\right),
$$

and further 


$$
E\left[z^{2}(t)\right]=\int_{0}^{t} \int_{0}^{t} d u d v \int_{0}^{\infty} \int_{0}^{\infty} \phi(x, y ; r) d x d y
$$

with $r=r(u-v)$.

For any fixed $r$ in $(-1,1)$ we have the identity

$$
\begin{aligned}
\int_{c}^{\infty} \int_{c}^{\infty} \phi(x, y ; r) d x d y & \\
& =\left(\int_{0}^{\infty} \phi(x) d x\right)^{2}+\frac{1}{2 \pi} \int_{0}^{r} \exp \left(-\frac{c^{2}}{1+w}\right) \frac{d w}{\left(1-w^{2}\right)^{1 / 2}} .
\end{aligned}
$$

(For $r=0$ the identity is obvious, and some calculation will show that the derivatives of both sides with respect to $r$ are equal.)

It then follows that the variance of $z(t)$ is

$$
\begin{aligned}
\operatorname{Var}[z(t)] & =\frac{1}{2 \pi} \int_{0}^{t} \int_{0}^{t} d u d v \int_{0}^{r(u-v)} \exp \left(-\frac{c^{2}}{1+w}\right) \frac{d w}{\left(1-w^{2}\right)^{1 / 2}} \\
& <\frac{1}{\pi^{2}} \int_{0}^{t} \int_{0}^{t}|r(u-v)| \exp \left(-\frac{c^{2}}{1+|r(u-v)|}\right) d u d v .
\end{aligned}
$$

From our assumptions concerning the spectral density $f(\lambda)$, it follows that there exist positive constants $k$ and $m$ such that

$$
\begin{array}{ll}
|r(t)|<\frac{k}{|t|} & \text { for all } t, \\
|r(t)| \leqq 1-m^{2} t^{2} & \text { for }|t| \leqq 2 k .
\end{array}
$$

(The latter inequality is easily proved by means of Cramér [4, Lemma 1].)

Dividing the domain of integration in (5) into two parts, defined respectively by $|u-v|>2 k$ and $|u-v| \leqq 2 k$, and using in each part the appropriate inequality for $|r(u-v)|$, we obtain from (5) by some straightforward estimation

$$
\operatorname{Var}[z(t)]<2 k t \log t \exp \left(-\frac{2 c^{2}}{3}\right)+\frac{2 \pi^{1 / 2}}{m} \cdot \frac{t}{c} \exp \left(-\frac{c^{2}}{2}\right)
$$

Now the Tchebychev inequality gives

$$
P[z(t)=0] \leqq \frac{\operatorname{Var}[z(t)]}{E^{2}[z(t)]} .
$$

\section{Taking}




$$
c=(2 \log t)^{1 / 2}-\frac{\log \log t}{(\log t)^{1 / 2}},
$$

we then obtain from (3), (4) and (6)

$$
P[\max x(u) \leqq c]<A\left((\log t)^{2} t^{-1 / 3}+(\log t)^{1 / 2-2^{1 / 2}}\right)
$$

where $A$ is independent of $t$. Since the second member obviously tends to zero as $t \rightarrow \infty$, (2) is proved.

3. It now remains to prove that

$$
P\left[\max x(u) \geqq(2 \log t)^{1 / 2}+\frac{\log \log t}{(\log t)^{1 / 2}}\right] \rightarrow 0
$$

as $t \rightarrow \infty$. For any $c>0$ we evidently have

$$
\begin{aligned}
P[\max x(u) \geqq c] & =P[\min x(u) \leqq c \leqq \max x(u)]+P[\min x(u)>c] \\
& =P_{1}+P_{2} .
\end{aligned}
$$

$P_{1}$ is, for any continuous sample function $x(u)$, the probability of at least one "crossing" with the level $c$ within $[0, t]$, i.e., the probability that there is at least one point $u$ in $[0, t]$ such that $x(u)=c$. Let $N$ denote the total number of such points, and write $p_{n}=P[N=n]$ for $n=0,1, \cdots$ Then

$$
P_{1}=p_{1}+p_{2}+\cdots \leqq p_{1}+2 p_{2}+\cdots=E[N] .
$$

However, it is known (Bulinskaya [3]) that under the present conditions

$$
E[N]=\frac{\left(\lambda_{2}\right)^{1 / 2}}{\pi} t \exp \left(-\frac{c^{2}}{2}\right)
$$

where $\lambda_{2}$ denotes the second moment of $f(\lambda)$. Further

$$
\begin{aligned}
P_{2} & =P[\min x(u)>c] \leqq P[x(0)>c] \\
& =\int_{0}^{\infty} \phi(x) d x<\frac{1}{c(2 \pi)^{1 / 2}} \exp \left(-\frac{c^{2}}{2}\right) .
\end{aligned}
$$

Taking now

$$
c=(2 \log t)^{1 / 2}+\frac{\log \log t}{(\log t)^{1 / 2}}
$$

it follows from (8), (9) and (10) that $P_{1}$ and $P_{2}$ both tend to zero as $t \rightarrow \infty$, so that (7) is proved. Finally, the result (1) follows from (2) and (7). 


\title{
REFERENCES
}

1. Yu. K. Belayev, Continuity and Hölder's conditions for sample functions of stationary Gaussian processes, Proc. Fourth Berkeley Symp., Vol. 2, pp. 23-33, 1961.

2. S. M. Berman, A law of large numbers for the maximum in a stationary Gaussian sequence, Ann. Math. Statist. 33 (1962), 93-97.

3. E. V. Bulinskaya, On the mean number of crossings of a level by a stationary Gaussian process, Teor. Verojatnost. i Primenen. 6 (1961), 474-477.

4. H. Cramér, Random variables and probability distributions, Cambridge Tracts in Mathematics, Vol. 36, Cambridge University Press, Cambridge, 1937. 2nd ed. to appear in 1962.

5. G. A. Hunt, Random Fourier transforms, Trans. Amer. Math. Soc. 71 (1951), 38-69.

6. M. Loève, Probability theory, 2nd ed., Van Nostrand, Princeton, N. J., 1960.

Research Triangle InStitute

\section{ON THE MAXIMUM TRANSFORM AND SEMIGROUPS OF TRANSFORMATIONS}

\author{
BY RICHARD BELLMAN AND WILLIAM KARUSH
}

Communicated by Peter D. Lax, April 27, 1962

1. Introduction. The problem of determining the maximum of the function

$$
F\left(x_{1}, x_{2}, \cdots, x_{N}\right)=\sum_{i=1}^{N} g_{i}\left(x_{i}\right)
$$

over the domain $D_{N}$ defined by $\sum_{i=1}^{N} x_{i}=x, x_{i} \geqq 0$, is one with various ramifications and applications. Analytic solutions and computational algorithms have been obtained in a number of ways; see Karush [7], Bellman [2], Bellman and Karush [3]. Let us now discuss a new way of generating solutions of (1.1). Let $g(x, a)$ be a scalar function of the scalar variable $x$ and the $M$-dimensional vector $a$ with the group property that

$$
\max _{x_{1}+x_{2}=x}\left[g\left(x_{1}, a\right)+g\left(x_{2}, b\right)\right]=g(x, h(a, b)) \quad\left(x_{1}, x_{2} \geqq 0\right),
$$

where $h(a, b)$ is a known function of $a$ and $b$. It follows inductively that

$$
\max _{D_{N}}\left[\sum_{k=1}^{N} g\left(x_{k}, a^{(k)}\right)\right]=g\left(x, h\left(a^{(1)}, a^{(2)}, \cdots, a^{(N)}\right)\right),
$$

where $D_{N}$ is as above, and $h\left(a^{(1)}, a^{(2)}, \cdots, a^{(N)}\right)$ is obtained from 